\title{
Combatiendo la Due Process Paranoia. Asegurando un ejercicio de la discrecionalidad arbitral con razonabilidad
}

\author{
Andrés Talavera Cano*** \\ Recibido/Received: 04/04/2017 \\ Aceptado/Accepted: 07/04/2017
}

SUMARIO: 1. Introducción. 2. La Due Process Paranoia hoy. 3. ¿Principal diagnóstico y cómo curarla? 3.1 Primer Paso: Identificar los problemas (Issues). 3.1.1 La parte que hace el pedido y su derecho a presentar su caso y ser oída 3.1.2 El derecho a que la disputa se resuelva de forma oportuna. 3.2 Segundo paso: Reglas aplicables (Rules); 3.2.1 Análisis de adecuación. 3.2.2 Análisis de necesidad. 3.2.3 Análisis de Proporcionalidad en sentido estricto. 3.3 Tercer paso: Aplicar el test (Analysis). 3.4 Cuarto paso: Conclusión del análisis (Conclusión). 4. Reflexiones finales.

PALABRAS CLAVE: Due Process Paranoia, Derecho de una parte a presentar su caso y ser oída, Derecho a que la controversia se resuelva de forma oportuna, Arbitraje, Test de proporcionalidad, Discrecionalidad de los árbitros.

KEYWORDS: Due Process Paranoia, right to be heard and present ones case, right to get the dispute solve in a timely manner, Arbitration, Proportionality Test.

* Asociado de Bullard, Falla \& Ezcurra Abogados, Profesor de Acto Jurídico, Contratos, Responsabilidad Civil, Seminario de Derecho Civil y Destrezas Legales en la Facultad de Derecho en la Universidad del Pacífico y Comunicación Jurídica Eficaz de la Facultad de Derecho de la Pontificia Universidad Católica del Perú. Abogado por la Pontificia Universidad Católica del Perú, «http://www.bullardabogados.pe/ne_atalavera.htm».

** El autor desea agradecer especialmente el apoyo de Julio Olórtegui en la preparación del presente artículo. Sin su apoyo, compromiso e investigación, su elaboración no hubiera sido posible.

A. Talavera Cano, "Combatiendo la Due Process Paranoia. Asegurando un ejercicio de la discrecionalidad arbitral con razonabilidad”, Revista Ecuatoriana de Arbitraje, No. 8, 2016. 
RESUMEN: En el presente artículo el autor sostiene que la principal problemática con la due process paranoia es la incertidumbre que tienen los árbitros cuando ejercen la discrecionalidad que les es reconocida en la toma de decisiones procesales, puesto que no tienen la certeza de que dichas decisiones puedan ser consideradas por los jueces como razonables y, en consecuencia, generar la anulación del Laudo por las cortes nacionales. Como "cura" para esta paranoia, el autor propone y desarrolla, paso por paso, un método lógico que servirá de guía para la toma de este tipo de decisiones arbitrales, y así se asegure su razonabilidad y validez de cara a los procesos de anulación de laudo frente a las cortes nacionales. Esta herramienta se ha creado sobre la base de la metodología IRAC y, en particular, la aplicación del test de proporcionalidad. En concreto, se buscan decisiones arbitrales que reflejen y concreticen la discrecionalidad arbitral con razonabilidad.

ABSTRACT: The author believes the main issue of the due process paranoia lays on the uncertainty arbitrators have regarding their procedural decisions, taking into consideration the award can be annuled if judges from national courts do not consider it is reasonable. As a "cure", the author proposes a logical solution based on the IRAC method and the proportionality test in order to give arbitral decisions validity and reasonability against possible actions of annulment.

\section{INTRODUCCIÓN}

Una historia cada vez más recurrente en los procesos arbitrales actuales es aquella en la que las partes abarrotan a los Tribunales con: (i) presentación de solicitudes de prórrogas de plazo; (ii) presentación de documentos después de la fecha límite establecida por los Tribunales Arbitrales; (iii) presentación de solicitudes de reprogramación de una audiencia a "último momento"; (iv) La presentación de una reclamación/pretensiones en el "úl- 
timo minuto"; y (v) Presentación de un escrito no solicitado, pero que a decir de las partes es "indispensable", entre otros.

La flexibilidad del arbitraje y el temor de verse enfrentados a un proceso que anule sus respectivos laudos, enfrenta a los árbitros al dilema de "otorgar o no otorgar" estos pedidos. A partir de las decisiones arbitrales que emitan sobre dichos pedidos, las partes podrán "sembrar" una posterior causal de anulación. En ese contexto, los árbitros quieren evitar que sus laudos sean anulados debido a que dichas decisiones arbitrales habrían vulnerado el derecho de defensa o el debido proceso, por un lado; pero, por el otro, saben que estos pedidos hacen que la decisión final se postergue y que quizás no llegue en debido tiempo y forma. Esta es la Due Process Paranoia que enfrentan los árbitros hoy en día y este artículo pretende proponer un proceso, paso a paso, a fin de analizar esos pedidos y decidir, caso por caso, si corresponde o no acceder a ellos.

El objetivo central: proponer un test que brinde predictibilidad en este tipo de decisiones a fin de que las cortes nacionales evalúen correctamente los cuestionamientos a las decisiones tomadas por los árbitros en estas circunstancias, como parte de los procesos de anulación de Laudo.

\section{La Due Process Paranoia hoy}

La creciente preocupación por la due process paranoia no es gratuita. Como señala la Queen Mary University of London y la firma White $\mathcal{E}$ Case, en su reporte sobre arbitraje internacional del $2015^{1}$ (en adelante, el Reporte del 2015):

One issue that merits special attention is the phenomenon that an interviewee dubbed "due process paranoia". This issue was repeatedly raised in responses, including in nearly all the personal interviews. "Due process paranoia" describes a reluctance by tribunals to act decisively in certain situations for fear of the arbitral award

1. Queen Mary University of London y White \& Case, 2015 International Arbitration Survey: Improvements and Innovations in International Arbitration, «https://goo.gl/Prj7iX». 


\section{Combatiendo la Due Process Paranoia. \\ Asegurando un ejercicio de la discrecionalidad arbitral con razonabilidad}

being challenged on the basis of a party not having had the chance

to present its case fully ${ }^{2}$.

Como se puede apreciar, los resultados son preocupantes. La gran mayoría de los entrevistados ${ }^{3}$. la mencionaron como uno de los principales problemas del arbitraje internacional hoy en día. Esto quiere decir que la due process paranoia es un fenómeno que ha calado profundamente en la mente de los árbitros.

Asimismo, esta paranoia ha tenido repercusiones y viene siendo reportada a nivel de la comunidad arbitral internacional ${ }^{4}$.

\section{3. ¿Principal diagnóstico y cómo CURARla?}

Como han señalado BERGER y JENSEN ${ }^{5}$, existen al menos cinco situaciones que, lamentablemente, no son extrañas a los procesos arbitrales, y que pueden gatillar en la mente de los árbitros esta paranoia:

(i) Un pedido de extensión de plazo;

(ii) La presentación de documentos fuera de la fecha acordada por las partes o fijada por el Tribunal;

(iii) La presentación de un escrito "sumamente importante" no establecido en el Calendario Procesal o bajo el acuerdo de las partes;

(iv) El pedido de reprogramación de una audiencia "a la última hora"; $y$,

(v) La inclusión a "último minuto" de un reclamo y/u pretensión.

2. Queen Mary University of London y White \& Case, N. 1.

3. De acuerdo a lo señalado en el Reporte del 2015, se entrevistó a 105 practicantes a nivel internacional.

4. E. Law, Due Process Paranoia, «https://goo.gl/aRvU4V» (06/06/2016). Véase, Freshfields Bruckhaus Deringer, «https://goo.gl/dHDNxc» (02/11/2016).

5. K. P. Berger y O. Jensen, "Due process paranoia and the Procedural Judgment Rule: A safe harbour for procedural management decisions by international arbitrators", Arbitration International, Vol. 32(3), 2016, pp. 415-435. 
¿Pero por qué estas situaciones generan tal paranoia? BERGER y JENSEN esbozan una explicación:

In some scenarios, this task is relatively easy to complete because the parties' procedural moves are either clearly legitimate exercises of their procedural rights, or 'guerrilla tactics', obviously motivated solely by the desire to obstruct the arbitral process. However, most cases are not that clear-cut. There are instances in which arbitrators find themselves in a 'grey zone' in which the true motives of the parties are not so easily discernible and the 'right' decision is not immediately apparent. In these situations, their procedural management decisions revolve around two crucial aspects of the arbitral process: the tribunal's quest for streamlined proceedings caused by the users' increasing demand for time -and cost-efficiency on one side; and the arbitrators' role as guardians of due process and guarantors of the legitimacy of arbitration on the other 6 .

Esta idea es complementada y clarificada por CAIVANO, quien comentando una de las causales de no reconocimiento de la Convención de Nueva York ${ }^{7}$ ha señalado lo siguiente:

Lo que en el fondo la norma postula, es que el razonable grado de discrecionalidad que los árbitros tienen en la conducción del procedimiento debe ser ejercido teniendo en mente que existe un umbral mínimo que no se puede transponer: ese umbral exige a los árbitros la necesidad de sopesar las garantías básicas de las partes, de modo de hallar un adecuado balance entre derecho de defensa y eficacia, dos valores igualmente capitales en el arbitraje ${ }^{8}$.

Lo dicho por CAIVANO es ilustrativo del problema que enfrentan los árbitros. Esta paranoia se crea debido a que, por un lado, los árbitros tienen en la cabeza su deber de emitir un laudo válido

6. Ibídem.

7. La causal comentada por CAIVANO es el artículo V.1b) de la Convención de Nueva York, causal que aborda la denegación de la ejecución / ejecución de un laudo por violación al debido proceso. Esta causal fue posteriormente adoptada con un texto bastante similar por la UNCITRAL en su Ley Modelo como una causal de anulación de laudo, por lo que ha sido también adoptada por todos los países que han basado sus normas en dicha Ley Modelo.

8. R. Caivano, Control Judicial en el Arbitraje, Abeledo Perrot, 2011, p. 348. 


\section{Combatiendo la Due Process Paranoia. \\ Asegurando un ejercicio de la discrecionalidad arbitral con razonabilidad}

y ejecutable ${ }^{9} y$, por el otro, no tienen certeza de si la decisión que tomen será considerada por las cortes, particularmente de anulación, como un uso razonable de su discrecionalidad en la conducción del procedimiento.

En la ley de arbitraje peruana, se esbozan algunos principios que guían el ejercicio de esa discrecionalidad, a saber:

- Tratar a las partes con igualdad y darle a cada una de ellas suficiente oportunidad de hacer valer sus derechos.

- A criterio del árbitro, ampliar los plazos que haya establecido para las actuaciones arbitrales, incluso si estos plazos estuvieran vencidos.

- En el curso de las actuaciones, cualquiera de las partes podrá modificar o ampliar su demanda o contestación, a menos que el árbitro considere que no corresponde permitir esa modificación en razón de la demora con que se hubiere hecho, el perjuicio que pudiera causar a la otra parte o cualesquiera otras circunstancias.

Por otro lado, en la referida ley, se regula una causal de anulación de laudo que es particularmente relevante al nacimiento de esta paranoia, aquella que señala que el laudo será anulado en caso el árbitro no haya permitido a la parte hacer valer sus derechos. Así de amplia y, por ende, así de incierta.

Las decisiones arbitrales que están vinculadas con este tipo de pedidos, suponen -en gran medida- el ejercicio de esa discrecionalidad. Sin embargo, si esa discrecionalidad termina "no permitiendo a la parte hacer valer sus derechos" podría generar la anulación del laudo.

9. D. Alessi, "Enforcing Arbitrator's Obligations: Rethinking International Commercial Arbitrators Liability", Journal of International Arbitration, Vol. 31, Issue 6, 2014, p. 765. "The final product of any arbitration is an enforceable final award. Enforceability of the award is the founding reason of the whole arbitration process. In order to render a valid and enforceable award, the arbitrator must comply with the following ancillary obligations: to follow the applicable formal requirements; to proceed in accordance with the parties' agreement or lex arbitri; and to observe the mandatory rules of the lex arbitri, as well as the rules of the likely place of enforcement". 
Entonces, ¿cómo logramos superar esta paranoia? Encontrando la forma de poder darles a los árbitros la seguridad de que la decisión que ellos tomen no será considerada como no razonable por las cortes nacionales y que no derivará en la anulación del laudo. En otras palabras, es necesario proponer un método lógico de toma de decisiones arbitrales, que permita ejercer esa discrecionalidad con razonabilidad.

¿Cómo logramos esto? Ante situaciones complejas, respuestas simples. Consideramos que la forma más sencilla y a la vez efectiva de analizar esta problemática es aplicando la metodología I.R.A.C. ¿En qué consiste esta metodología? En seguir un proceso lógico conformado por cuatro pasos que nos aseguran tener una decisión razonable: (i) el Primero, identificar los problemas o Issues; (ii) el segundo, identificar las reglas aplicables o Rules; (iii) el tercero, aplicar las reglas a los hechos o Analysis, y; (iv) llegar a una conclusión o Conclusion.

A continuación, desarrollaremos la aplicación de esta metodología al problema en cuestión:

\subsection{Primer paso: Identificar el problema (Issues)}

El primer paso para aplicar esta metodología es identificar cuál es el problema que tienen que enfrentar los árbitros al momento de decidir respecto a una de las situaciones presentadas por BERGER Y JENSEN.

Para esto, debemos revisar la posición de las partes en la disputa:

\subsubsection{La parte que hace el pedido y su derecho a presentar su caso y ser oída}

Todos los casos descritos por BERGER y JENSEN tienen como fundamento este derecho. De acuerdo a AlONSO: 


\section{Combatiendo la Due Process Paranoia. \\ Asegurando un ejercicio de la discrecionalidad arbitral con razonabilidad}

El derecho de una parte a presentar su caso o a ser oído, también llamado principio de contradicción, es el corolario del derecho de defensa y significa que las partes de un procedimiento deberán tener la oportunidad de presentar su posición antes de que se dicte una decisión ${ }^{10}$.

En este sentido, al momento de, por ejemplo, decidir si aceptar o no la presentación de un nuevo reclamo casi a fin del arbitraje, el Tribunal Arbitral deberá ponderar si es que el rechazar dicha solicitud impediría a una parte a presentar su caso o exponer sus argumentos.

En el Perú, sin perjuicio de sus raíces constitucionales, este derecho se encuentra recogido en el artículo 34 de la Ley de Arbitraje $\mathrm{e}^{11}$, teniendo como base el artículo $18^{12}$ de la Ley Modelo UNCITRAL, derecho considerado por sus redactores como la carta magna del arbitraje internacional, al ser un límite para la facultad del Tribunal Arbitral de dirigir el proceso $^{13}$.

\subsubsection{El derecho a que la disputa se resuelva de forma oportuna}

Por otro lado, y en contraposición con el derecho antes mencionado, se encuentra el derecho de las partes a que su disputa se resuelva de forma oportuna y evitando dilaciones indebidas. Al respecto, el Tribunal Constitucional Peruano ha señalado que:

En relación al derecho a ser juzgado sin dilaciones indebidas, este Tribunal considera pertinente recordar que el derecho a que una persona sea juzgada dentro de un plazo razonable

10. J.M. Alonso, "El derecho de defensa", en G. TAwIl y E. Zuleta (Eds.), El Arbitraje Comercial Internacional. Estudio de la Convención de Nueva York con motivo de su $50^{\circ}$ aniversario, Abeledo Perrot, 2008, p. 456.

11. Ley de Arbitraje, Decreto Legislativo 1071, Art. 34: Libertad de regulación de actuaciones “(...) 2. El tribunal arbitral deberá tratar a las partes con igualdad y darle a cada una de ellas suficiente oportunidad de hacer valer sus derechos".

12. Ley Modelo UNCITRAL, Art. 18: Trato equitativo de las partes "Deberá tratarse a las partes con igualdad y darse a cada una de ellas plena oportunidad de hacer valer sus derechos".

13. H. Holtzmann y J. Neuhaus, A Guide to the UNCITRAL Model Law on International Commercial Arbitration: Legislative History and Commentary, Kluwer Law International, 1989. 
no se encuentra expresamente contemplado en la Constitución. Sin embargo, se trata de un derecho que coadyuva el pleno respeto de los principios de proporcionalidad, razonabilidad, subsidiariedad, necesidad, provisionalidad y excepcionalidad, que debe guardar la duración de un proceso para ser reconocido como constitucional. Se trata, propiamente, de una manifestación implícita del derecho al debido proceso y la tutela judicial efectiva reconocidos en la Carta Fundamental (artículo $139^{\circ} 3$ de la Constitución) y, en tal medida, se funda en el respeto a la dignidad de la persona humana ${ }^{14}$.

Por ejemplo, tomando el caso antes mencionado, de aceptar el Tribunal Arbitral el nuevo reclamo presentado por una de las partes, esto traería consigo que se tenga que modificar el Calendario Procesal para otorgarle a la otra parte un plazo razonable para que esta responda las nuevas alegaciones. Esto también podría dar pie a que se celebren nuevas audiencias en caso que la discusión sea muy complicada y el Tribunal Arbitral estimase prudente oír a las partes. Todo lo anterior, podría retrasar significativamente la emisión del laudo arbitral, lo cual traería consigo perjuicios para las partes tanto desde un punto de vista de justicia como comercial.

En el Perú, como señalamos anteriormente, la jurisprudencia enmarca este derecho como un derivado del derecho al debido proceso y a la tutela jurisdiccional efectiva ${ }^{15}$. Asimismo, debemos señalar que en los últimos años este viene teniendo un mayor reconocimiento a nivel internacional, lo cual se puede apreciar en las mayores medidas a nivel institucional que se están tomando,

14. Tribunal Constitucional del Perú, Luisa Jáuregui Villanueva c. Segunda Sala Penal Para Procesos con Reos Libres de la Corte Superior de Justicia de Lima, Expediente N 549-2004-HC/TC, 21/01/2005.

15. Tribunal Constitucional del Perú, Luisa Jáuregui Villanueva c. Segunda Sala Penal Para Procesos con Reos Libres de la Corte Superior de Justicia de Lima, Expediente No 549-2004-HC/TC, 21/01/2005. Véase, Tribunal Constitucional del Perú, Juan Humberto Quiroz Rosas c. Cuarta Sala Civil de la Corte Superior de Justicia de Lima, Expediente N²732-2007-PA/TC, 05/11/2007. “(...) en el artículo III del Código Procesal Constitucional obligan a este Tribunal a pronunciarse respecto a la importancia del derecho al debido proceso en su manifestación del derecho a un plazo razonable en la administración de justicia, que si bien es cierto, y como ha quedado dicho, no constituye el fondo de la controversia, ni ha sido denunciado por el recurrente, ha sido directamente comprometido por los operadores judiciales al presentarse una serie de, por decir lo menos, irregularidades o situaciones anómalas que en conjunto permiten considerar una indiscutible vulneración del citado atributo". 
sobre todo por la CCI, para promover la celeridad y la eficiencia en el proceso arbitral ${ }^{16}$.

En este sentido, tenemos que al momento de resolver un pedido como el mencionado párrafos arriba, el Tribunal Arbitral tendrá al frente un conflicto entre dos derechos: (i) por un lado, el derecho de la parte que solicita la medida a poder presentar su caso y ser oída; y, (ii) por el otro, el derecho de la otra parte a que su disputa sea resuelta de forma oportuna y sin dilaciones indebidas.

Ante esto, el Tribunal Arbitral debe identificar cual será el set de reglas más adecuadas para poder resolver este conflicto.

\subsection{Segundo paso: Reglas aplicables (Rules)}

Habiendo determinado que el problema en estos casos es uno de conflicto entre derechos, puntualmente, dos derechos derivados del derecho a un debido proceso, el Tribunal Arbitral deberá utilizar una herramienta que le permita abordar esta disyuntiva de la forma más adecuada.

A nivel de doctrina constitucional, cuando las cortes se ven ante la necesidad de analizar un conflicto como el presente, recurren a una herramienta denominada el test de proporcionalidad o ponderación de derechos el cual permite analizar los límites de dos derechos que colisionan entre sí. Así, de acuerdo a VIDAL:

(...) resulta fundamental la teoría de los límites inmanentes de los derechos fundamentales, que se construye a partir de la reiterada afirmación de nuestro Tribunal Constitucional de que todo derecho fundamental tiene sus límites, que en ocasiones aparecen expresamente previstos en el texto constitucional, pero que en otras derivan de una manera mediata o indirecta de tal norma "en cuanto han de justificarse por la

16. International Chamber of Commerce, ICC Court announces new policies to foster transparency and ensure greater efficiency, «https://goo.gl/LbAz7m» (12/11/2016). Véase, International Chamber of Commerce, ICC Court amends its Rules to enhance transparency and efficiency, «https://goo.gl/KWv77u» (12/11/2016). 
necesidad de proteger o preservar no sólo otros derechos constitucionales, sino también otros bienes constitucionalmente protegidos. (Sentencia del Tribunal Constitucional español [STC] 2/82, entre otras muchas $)^{17}$.

En este sentido, esta herramienta sirve para ponderar y medir los límites de aquellos derechos fundamentales (en este caso, dos derechos derivados del debido proceso). La aplicación de esta herramienta, de raíces constitucionales, permitirá a los árbitros fundamentar lógicamente el ejercicio de su discrecionalidad con razonabilidad. Debe tenerse en cuenta que el test de proporcionalidad deberá matizarse para su aplicación en los procesos arbitrales, tenerlo como línea guía a fin de que sirva de método lógico para esta toma de decisiones. Con esto claro, ¿cuáles son los pasos a seguir para utilizar este test? La doctrina ha determinado que su aplicación se encuentra supeditada a tres pasos:

\subsubsection{Análisis de adecuación}

Este análisis "exige la adecuación medio-fin en que toda restricción de un derecho fundamental se estructura; (...) es necesario que la limitación que sufre el derecho resulte apropiada para lograr el fin que lo justifica"18.

En sede nacional, el Tribunal Constitucional Peruano ha señalado que este análisis supone "(...) de un lado, que ese objetivo sea legítimo; $y$, de otro, que la idoneidad de la medida examinada tenga relación con el objetivo, es decir, que contribuya de algún modo con la protección de otro derecho o de otro bien jurídico relevante" 19 .

17. C. VIDAL, "El principio de proporcionalidad como parámetro de constitucionalidad de la actividad del juez", Anuario de Derecho Constitucional Latinoamericano 2005, «https://goo.gl/4DvyNW» $(13 / 11 / 2016)$.

18. C. VIDAL, N. 17.

19. A. Burga, "El test de ponderación o proporcionalidad de los derechos fundamentales en la jurisprudencia del Tribunal Constitucional peruano", Gaceta Constitucional No 47, Gaceta Jurídica, 2011. Citando a Tribunal Constitucional Peruano, 5186 ciudadanos contra los Decretos Legislativos 921, 922, 923, 924, 925, 926 y 927, Expediente $\mathrm{N}^{\circ}$ 003-2005-PI/TC, 09/08/2006. 
En el caso particular, para enmarcar la decisión del Tribunal Arbitral dentro de un manto de razonabilidad y así evitar el due process paranoia, el Tribunal Arbitral no analizará en sí la idoneidad de la decisión que vaya a tomar, sino la idoneidad de lo solicitado por la parte actora. Con esto en claro, consideramos que la pregunta al momento de realizar el análisis de idoneidad es la siguiente:

- ¿La solicitud presentada por una de las partes es adecuada para el fin que busca?

En caso esta pregunta sea respondida de forma afirmativa, el Tribunal Arbitral deberá continuar con el siguiente paso del test.

\subsubsection{Análisis de Necesidad}

El segundo paso del test es someter la medida a un análisis de necesidad, según el cual "no ha de existir 'otra medida limitadora igualmente efectiva pero de menor incidencia en el derecho fundamental de los afectados ${ }^{\prime \prime \prime 20}$.

En sede nacional, el Tribunal Constitucional Peruano ha señalado que:

Bajo este test ha de analizarse si existen medios alternativos al optado por el legislador que no sean gravosos o, al menos, que lo sean en menor intensidad. Se trata del análisis de una relación medio-medio, esto es, de una comparación entre medios; el optado por el legislador y el o los hipotéticos que hubiera podido adoptar para alcanzar el mismo fin. Por esto, el o los medios hipotéticos alternativos han de ser igualmente idóneos ${ }^{21}$.

De esta forma, al aplicar este análisis al caso en concreto, el Tribunal Arbitral deberá analizar si la parte actora contaba con alguna alternativa menos gravosa para la otra parte que las soli-

20. C. VidaL, N. 17.

21. Tribunal Constitucional Peruano, Colegio de Abogados de Lima contra el artículo 3 de la Ley $N^{a} 27466$, Expediente N ${ }^{\mathrm{a}}$ 045-2004-PI/TC, 29/10/2005. 
citadas. Ante esto, proponemos las siguientes preguntas como punto de partida para el análisis del Tribunal Arbitral.

- ¿Es la única alternativa y la menos costosa para lograr lo que la parte está buscando con su petición?

- ¿Sólo ahora podía optar por esa alternativa?

Solo en aquellos casos donde estas preguntas hayan sido respondidas de forma afirmativa se podrá pasar al siguiente nivel de análisis.

\subsubsection{Análisis de Proporcionalidad en sentido estricto}

Finalmente, en caso se cumplan con los dos puntos antes señalados, el tercer análisis que ha de realizarse está relacionado con si "los medios elegidos [mantienen] una relación razonable con el resultado perseguido" 22 .

En sede nacional, el Tribunal Constitucional Peruano ha senalado que:

La proporcionalidad en sentido estricto o ponderación (Abwägung), (...) consistirá en una comparación entre el grado de realización u optimización del fin constitucional y la intensidad de la intervención en la igualdad. La comparación de estas dos variables ha de efectuarse según la denominada ley de ponderación. Conforme a ésta: Cuanto mayor es el grado de la no satisfacción o de la afectación de un principio, tanto mayor tiene que ser la importancia de la satisfacción del otro $^{23}$.

Así, para ejecutar este análisis, el Tribunal Arbitral deberá responder dos preguntas relacionadas a la proporcionalidad de la medida solicitada por la parte actora:

- Si se concediera lo solicitado, ¿sería más beneficioso para el proceso y la solución de la controversia?

22. C. Vidal, N. 17.

23. Tribunal Constitucional Peruano, N. 21. 
- Si se concediera lo solicitado, ¿la limitación del derecho de la otra parte es razonable y no sería excesivamente gravosa?

\subsection{Tercer paso: Aplicar el test (Analysis)}

Habiendo determinado cuales son las "reglas aplicables" o, en este caso, la metodología y las preguntas que el Tribunal Arbitral deberá responder para lidiar con la due process paranoia, deberá aplicarse dichas reglas a cada caso en concreto, considerando los hechos y circunstancias particulares que enfrentan.

En otras palabras, el Tribunal Arbitral deberá realizar el "test de proporcionalidad" paso a paso, analizando las circunstancias del caso concreto, derivando las respuestas a cada una de las preguntas antes planteadas.

\subsection{Cuarto paso: Conclusión del análisis (Conclusion)}

Habiendo realizado el análisis anterior, el Tribunal Arbitral deberá decidir de acuerdo a los resultados obtenidos. En caso todas las preguntas antes propuestas sean respondidas de forma afirmativa, el Tribunal Arbitral deberá conceder la medida solicitada, siempre que esta cumple con el test de proporcionalidad, por lo que existe justificación para limitar el derecho de la otra parte.

Por el contrario, en caso alguna de las preguntas antes presentadas no fuese respondida de forma afirmativa, el Tribunal Arbitral tendrá justificadas razones para rechazar la solicitud presentada, pudiendo, en caso no hacerlo, vulnerar injustificadamente y sin un adecuado margen de razonabilidad el derecho de la parte que no solicita la medida. 


\section{REFLEXIONES FiNALES}

Frente al impacto que ha tenido la due process paranoia en la comunidad arbitral internacional, se deben proponer soluciones para lidiar con esta o "curarla". El presente artículo busca proponer, tomando como base la metodología IRAC y el test de proporcionalidad, como herramientas que ayuden a los árbitros a reducir al mínimo las razones por las cuales las cortes nacionales, especialmente de anulación, podrían considerar no razonable la decisión tomada por ellos, reduciendo así el grado de incertidumbre y borrando de sus mentes esta paranoia.

Consideramos que este método lógico puede ser utilizado por la parte que plantea "justificadamente" estas solicitudes, a fin de fundamentar y dotar de legitimidad su pedido. Igualmente, puede ser utilizado por la parte que cuestionará el otorgamiento de la medida, a fin de demostrar al Tribunal Arbitral que conceder la medida sería vulnerar injustificadamente sus derechos.

Finalmente y como un evidente aporte práctico, hemos incluido en el presente artículo una pequeña tabla de análisis denominada Test de Proporcionalidad aplicado a Procesos Arbitrales que esperemos pueda ser utilizado por los árbitros y operadores arbitrales para combatir esta paranoia. 


\section{Test de Proporcionalidad aplicado a Procesos Arbitrales}

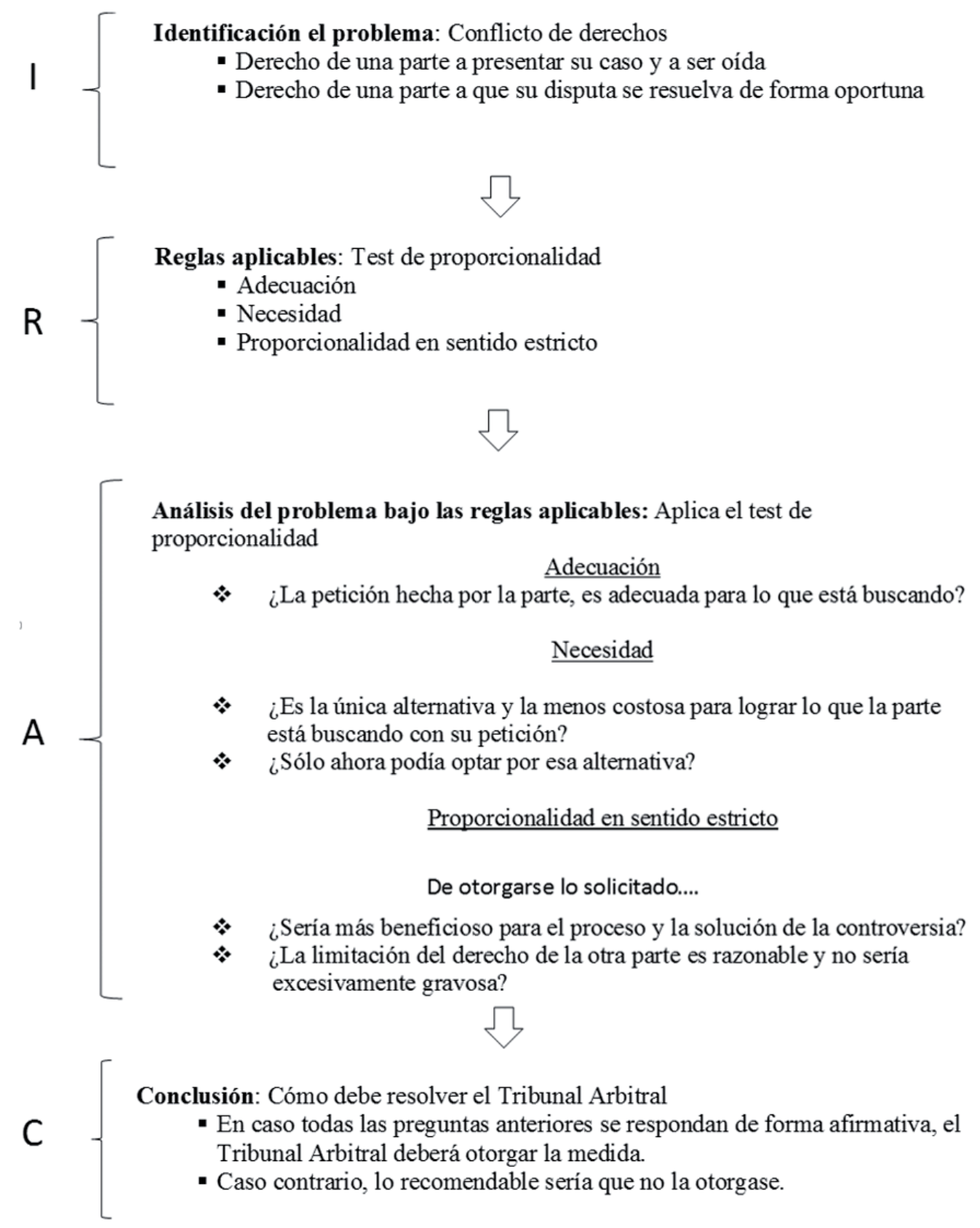

